\title{
REMARKS ON BESOV SPACES AND BEST POLYNOMIAL APPROXIMATION
}

\section{Z. DITZIAN AND V. TOTIK}

(Communicated by Richard R. Goldberg)

\begin{abstract}
Besov-type spaces that are generated by new moduli of smoothness are related to sequence spaces of $\left\{E_{n}(f)\right\}$ where $E_{n}(f)$ is the appropriate best algebraic polynomial approximation.
\end{abstract}

1. Introduction. The classical moduli of smoothness $\omega(f, t)_{p}$ are given for $f \in L_{p}(D)$ by

$$
\omega^{r}(f, t)_{p}=\sup _{0<h \leq t}\left\|\Delta_{h}^{r} f\right\|_{L_{p}(D)}
$$

where

$$
\Delta_{h}^{r} f(x)= \begin{cases}\sum_{k=0}^{r}\left(\begin{array}{l}
r \\
k
\end{array}\right)(-1)^{k} f(x+(k-r / 2) h) & \text { if }(x-r h / 2, x+r h / 2) \subset D \\
0 & \text { otherwise }\end{cases}
$$

The related Besov spaces are given by their norms (see [1]).

$$
\|f\|_{B_{\alpha, s}^{p}}=\|f\|_{p}+\left\|t^{-\alpha} \omega^{r}(f, t)_{p}\right\|_{L_{s}^{*}}
$$

where

$$
\|g\|_{L_{s}^{*}}= \begin{cases}\left\{\int_{0}^{1}|g(t)|^{s} t^{-1} d t\right\}^{1 / s} & \text { for } 1 \leq s<\infty \\ \sup _{t}|g(t)| & \text { for } s=\infty\end{cases}
$$

for $0<\alpha<r$ and $1 \leq p \leq \infty$. It can be noted that, because of the term $\|f\|_{p}$ in $(1.3),(0,1)$ can be replaced by $(0, \infty)$ in $(1.4)$.

One of the interesting results about Besov spaces is that for periodic functions of period $2 \pi$ (that is, $D=T$ ) we have an equivalent norm to $\|f\|_{B_{a, s}^{p}}$ given by

$$
\|f\|_{B_{\alpha, s}^{p}}^{*} \equiv\left\|(n+1)^{\alpha} E_{n}^{*}(f)_{p}\right\|_{l_{s}^{*}}
$$

where for a sequence $\left\{a_{n}\right\}_{n=0}^{\infty}$

$$
\left\|\left\{a_{n}\right\}\right\|_{l_{s}^{*}}= \begin{cases}\left\{\sum_{n=0}^{\infty}\left|a_{n}\right|^{s}(n+1)^{-1}\right\}^{1 / s} & \text { for } 1 \leq s<\infty \\ \sup _{n}\left|a_{n}\right| & \text { for } s=\infty\end{cases}
$$

and where

$$
\begin{array}{r}
E_{n}^{*}(f)_{p}=\inf \left(\|f-\tau\|_{L_{p}(T)}: \tau \text { trigonometric polynomial }, \operatorname{deg} \tau<n\right) \\
\left(E_{0}^{*}(f)_{p}=\|f\|_{p}\right) .
\end{array}
$$

Received by the editors October 9, 1987.

1980 Mathematics Subject Classification (1985 Revision). Primary 41A10, 41A17, 41A27; Secondary $26 \mathrm{~A} 16$. 
The above can be shown as a corollary of Jackson's inequality

$$
E_{n}^{*}(f)_{p} \leq M \omega^{r}(f, 1 / n)_{p} \text { for } n>r
$$

and Stechkin's inequality

$$
\omega^{r}(f, 1 / n)_{p} \leq A_{r} n^{-r} \sum_{\nu=0}^{n}(\nu+1)^{r-1} E_{\nu}^{*}(f)_{p}
$$

(see [6, p. 235]). In a more general framework, analogues of the equivalence between (1.3) and (1.5) were given in [2 and 4]. In these articles, the key to the proof is the use of Jackson and Bernstein-type inequalities and the equivalence between the $K$-functional and the modulus of smoothness. We emphasize here the role of weak-type inequalities, that is, the analogue of (1.9), a weak Jackson inequality (see (3.9) for instance) and a weak relation between $K$-functional and the modulus of smoothness (see (5.7) for instance). This is useful as analogues of the equivalence between (1.3) and (1.5) are valid even in cases in which the analogue of Jackson's inequality is not true (see $\S \S 3$ and 5 ), the analogue of Bernstein's inequality was not stated being somewhat messy (see $\S 4$ ) or the $K$-functional is not equivalent to the modulus of smoothness discussed (see $\S \S 3$ and 5).

Other moduli of smoothness defined in [5] and related to best algebraic approximation in $L_{p}[-1,1]$ or in $L_{p}(S)$, where $S$ is a simple polytope in $R^{d}$, give rise to new Besov-type spaces and to results analogous to the equivalence between (1.3) and (1.5), since the analogues of (1.8) and (1.9) were already proved in [5]. Moreover, it will be shown that an expression defined in [5] and called "main-part moduli of smoothness", which is essential in investigating best-weighted polynomial approximation in $L_{p}[-1,1]$, still leads to equivalence between norms analogous to (1.3) and (1.5). What is special about this result is that the main-part moduli are not equivalent to the corresponding $K$-functionals and we do not have the analogue of Jackson's inequality for these moduli. The theorem achieved (Theorem 3.1) can be construed as yet one more result confirming the importance and usefulness of the main-part moduli.

Analogues for best polynomial approximation on $L_{p}(R)$ with the Freud weight $W_{\lambda}(x)=\exp \left(-|x|^{\lambda}\right)$ are also achieved.

We should stress that the key inequalities used here, i.e. (2.6), (2.7), (3.8), (3.9), (4.2), (5.8) and (5.9) of this paper, were achieved in our earlier work [5].

Equivalence between two expressions, for example norms, functions, sequences, integrals and sums, will be denoted by $\sim$ and will mean that each is smaller than a constant times the other (and the constant is independent of the domain, class of functions, class of sequences, etc.).

2. Besov spaces and best polynomial approximation. It was shown in $\left[5\right.$, Chapter 7] that to investigate best algebraic polynomial approximation $E_{n}(f)_{p}$ given by

$$
E_{n}(f)_{p}=\inf \left(\|f-P\|_{L_{p}[-1,1]} ; P \text { is an algebraic polynomial, } \operatorname{deg} P<n\right),
$$

$\left(E_{0}(f)_{p}=\|f\|_{p}\right)$, the appropriate tools are the moduli of smoothness given by

$$
\omega_{\varphi}^{r}(f, t)_{p}=\sup _{0<h \leq t}\left\|\Delta_{h \varphi}^{r} f\right\|_{L_{p}[-1,1]}, \quad \varphi(x)=\sqrt{1-x^{2}},
$$


where $\Delta_{\eta}^{r} f(x)$ is given by $(1.2)$ with $D=[-1,1]$. (Note that in (2.2) the increment $h \varphi$ depends on $x$.) We now have new Besov-type spaces and equivalent norms analogous to those given in (1.3) and (1.5).

THEOREM 2.1. For $0<\alpha<r, 1 \leq p \leq \infty$ and $1 \leq 3 \leq \infty$ the norms

$$
\|f\|_{B_{\alpha, s}^{p}(\varphi)}=\|f\|_{L_{p}[-1,1]}+\left\|t^{-\alpha} \omega_{\varphi}^{r}(f, t)_{p}\right\|_{L_{s}^{*}}
$$

and

$$
\|f\|_{B_{\alpha, s}^{p}(\varphi)}^{*}=\left\|(n+1)^{\alpha} E_{n}(f)_{p}\right\|_{l_{s}^{*}}
$$

are equivalent.

We remark that the Peetre $K$-functional

$$
K_{r, \varphi}\left(f, t^{r}\right)_{p}=\inf _{g^{(r-1)} \in \text { A.C. } \mathrm{loc}}\left(\|f-g\|_{L_{p}[-1,1]}+t^{r}\left\|\varphi^{r} g^{(r)}\right\|_{L_{p}[-1,1]}\right)
$$

was shown in [5] to be equivalent to $\omega_{\varphi}^{r}(f, t)_{p}$, and therefore,

$$
\|f\|_{B_{\alpha, s}^{p}(\varphi)}^{* *} \equiv\|f\|_{L_{p}[-1,1]}+\left\|t^{-\alpha} K_{r, \varphi}\left(f, t^{r}\right)_{p}\right\|_{L_{s}^{*}} \sim\|f\|_{B_{\alpha, s}^{p}(\varphi)} .
$$

ProOF. As $E_{n}(f)_{p}$ is decreasing in $n$, we have

and

$$
\sum_{0 \leq n \leq 2^{m}}(n+1)^{r-1} E_{n}(f)_{p} \sim \sum_{\nu=0}^{m} 2^{\nu r} E_{2^{\nu}}(f)_{p} \quad \text { for all } m \text { and } f
$$

$$
\sum_{n=1}^{\infty}\left((n+1)^{\alpha} E_{n}(f)_{p}\right)^{s}(n+1)^{-1} \sim \sum_{k=0}^{\infty}\left(2^{k \alpha} E_{2^{k}}(f)_{p}\right)^{s} \quad \text { for all } f .
$$

As $\omega_{\varphi}^{r}(f, t)_{p}$ is increasing in $t$, we have

$$
\int_{0}^{1}\left[t^{-\alpha} \omega_{\varphi}^{r}(f, t)_{p}\right]^{s} t^{-1} d t \sim \sum_{m=1}^{\infty}\left(2^{m \alpha} \omega_{\varphi}^{r}\left(f, 2^{-m}\right)_{p}\right)^{s} \quad \text { for all } f .
$$

We now use the Jackson-type inequality

$$
E_{n}(f)_{p} \leq L \omega_{\varphi}^{r}(f, 1 / n)_{p} \text { for } n>r
$$

which was recently proved [5, Theorem 7.2.1], and $E_{n}(f)_{p} \leq\|f\|_{p}$ for $n \leq r$, to obtain $\|f\|_{B_{\alpha, s}^{p}(\varphi)}^{*} \leq M\|f\|_{B_{\alpha, s}^{p}(\varphi)}$.

To prove $\|f\|_{B_{\alpha, s}^{p}(\varphi)} \leq M\|f\|_{B_{\alpha, s}^{p}(\varphi)}^{*}$, we recall the Stechkin-type inequality

$$
\omega_{\varphi}^{r}(f, t)_{p} \leq L t^{r} \sum_{0 \leq n \leq 1 / t}(n+1)^{r-1} E_{n}(f)_{p}
$$

proved recently [5, Theorem 7.2.4]. Using (2.7) and the equivalences above, we now have for $\alpha<r$

$$
\begin{aligned}
\int_{0}^{1}\left(t^{-\alpha} \omega_{\varphi}^{r}(f, t)_{p}\right)^{s} t^{-1} d t & \leq C \sum_{m=1}^{\infty}\left(2^{m \alpha} \omega_{\varphi}^{r}\left(f, 2^{-m}\right)_{p}\right)^{s} \\
& \leq C_{1} \sum_{m=1}^{\infty}\left(2^{m(\alpha-r)} \sum_{\nu=0}^{m} 2^{\nu r} E_{2^{\nu}}(f)_{p}\right)^{s} \\
& \leq C_{1} \sum_{m=1}^{\infty}\left\{2^{m(\alpha-r)} \sum_{\nu=0}^{m} 2^{\nu(r-\alpha)}\left(2^{\nu \alpha} E_{2^{\nu}}(f)_{p}\right)\right\}^{s} \equiv I .
\end{aligned}
$$


Using Hölder's inequality (or Jensen's inequality), we have

$$
\begin{aligned}
I & \leq C_{2} \sum_{m=1}^{\infty} 2^{m(\alpha-r)} \sum_{\nu=0}^{m} 2^{\nu(r-\alpha)} 2^{\nu s \alpha} E_{2^{\nu}}(f)_{p}^{s} \\
& \leq C_{2} \sum_{\nu=0}^{\infty} 2^{\nu s \alpha} E_{2^{\nu}}(f)_{p}^{s}\left\{\sum_{m=\nu}^{\infty} 2^{m(\alpha-r)}\right\} 2^{\nu(r-\alpha)} \\
& \leq C_{3} \sum_{\nu=0}^{\infty} 2^{\nu \alpha s} E_{2^{\nu}}(f)_{p}^{s} \leq M\left(\|f\|_{B_{\alpha, s}^{p}(\varphi)}^{*}\right)^{s} .
\end{aligned}
$$

Observing that $E_{0}(f)_{p}=\|f\|_{p}$, we complete the proof.

The above very simple computation was performed in almost excessive detail so that we can refer later in the paper to this type of argument.

3. Main part moduli and weighted polynomial approximation. Investigation of best weighted algebraic polynomial approximation

$$
E_{n}(f)_{w, p}=\inf \left(\|w(f-p)\|_{L_{p}[-1,1]} ; \operatorname{deg} P<n\right)
$$

where $w \in J_{p}^{*}$ as given in [5], a class which contains the Jacobi weights, that is $w(x)=(1-x)^{\gamma_{1}}(1+x)^{\gamma_{2}}$ for $\gamma_{i}>-1 / p$ was carried out in [3, Chapter 8]. This required the notion of weighted main-part moduli of smoothness given by

$$
\Omega_{\varphi}^{r}(f, t)_{w, p}=\sup _{0 \leq h \leq t}\left(\left\|w \Delta_{h \varphi}^{r} f\right\|_{L_{p}\left[-1+2 r^{2} h^{2}, 1-2 r^{2} h^{2}\right]} ; \varphi(x)=\sqrt{1-x^{2}}, w(x) \in J_{p}^{*}\right) .
$$

The corresponding $K$-functional is given by

$$
K_{r, \varphi}\left(f, t^{r}\right)_{w, p}=\inf _{g^{(r-1)} \in \text { A.C.loc }}\left(\|w(f-g)\|_{L_{p}[-1,1]}+t^{r}\left\|w \varphi^{r} g^{(r)}\right\|_{L_{p}[-1,1]}\right) .
$$

It was shown in [5] that $K_{r, \varphi}\left(f, t^{r}\right)_{w, p}$ is not equivalent to $\Omega_{\varphi}^{r}(f, t)_{w, p}$ even if $w=1$. However, $\Omega_{\varphi}^{r}$ can be defined for weights $w$ for which we cannot define $\omega_{\varphi}^{r}$ at all, it is easier to compute, and (see [5, Corollary 8.2.5]) it satisfies

$$
\Omega_{\varphi}^{r}(f, t)_{w, p}=O\left(t^{\alpha}\right) \Leftrightarrow K_{\varphi, r}\left(f, t^{r}\right)_{w, p}=O\left(t^{\alpha}\right)
$$

for $\alpha<r$. We may now state and prove the following theorem.

THEOREM 3.1. For $w \in J_{p}^{*}, 0<\alpha<r, 1 \leq s \leq \infty$ and $1 \leq p \leq \infty$ the norms

$$
\begin{gathered}
\|f\|_{B_{\alpha, s}^{p}(\varphi, w)}=\|w f\|_{p}+\left\|t^{-\alpha} K_{r, \varphi}\left(f, t^{r}\right)_{w, p}\right\|_{L_{s}^{*}} \\
\|f\|_{B_{\alpha, s}^{p}(\varphi, w)}^{*}=\|w f\|_{p}+\left\|t^{-\alpha} \Omega_{\varphi}^{r}(f, t)_{w, p}\right\|_{L_{s}^{*}}
\end{gathered}
$$

and

$$
\|f\|_{B_{\alpha, s}^{p}(\varphi, w)}^{* *}=\left\|(n+1)^{\alpha} E_{n}(f)_{w, p}\right\|_{l_{s}^{*}}
$$

are equivalent.

PROOF. For $s=\infty$ our theorem was proved in Corollary 8.2.2 and Corollary 8.2.5 of [5]. Following the proof Theorem 2.1 and the inequality

$$
\Omega_{\varphi}^{r}(f, t) \leq M t^{r} \sum_{0 \leq n \leq 1 / t}(n+1)^{r-1} E_{n}(f)_{w, p}
$$


given in Theorem 8.2.1 of [5], we have

$$
\|f\|_{B_{\alpha, s}^{p}(\varphi, w)}^{*} \leq M\|f\|_{B_{a, s}^{p}(\varphi, w)}^{* *}
$$

The direction $\|f\|^{* *} \leq M\|f\|^{*}$ that was easy in the proof of Theorem 2.1 now involves the inequality

$$
E_{n}(f)_{w, p} \leq A \sum_{k=0}^{\infty} \Omega_{\varphi}^{r}\left(f, n^{-1} 2^{-k}\right)_{w, p}, \quad n>r
$$

which is again part of Theorem 8.2.1 of [5]. As $\Omega_{\varphi}^{r}(f, t)_{w, p}$ increases with $t$, and $E_{n}(f)_{w, p}$ decreases with $n$, we have for $0<\delta<\alpha$

$$
\begin{aligned}
\left(\|f\|_{B_{\alpha, s}^{p}(\varphi, w)}^{* *}\right)^{s} \leq B \sum_{t=0}^{\infty}\left(2^{l \alpha} E_{2^{l}}(f)_{w, p}\right)^{s} \\
\quad \leq B A \sum_{l=0}^{\infty} 2^{l s \alpha}\left\{\sum_{k=l}^{\infty} \Omega_{\varphi}^{r}\left(f, 2^{-k}\right)_{w, p}\right\}^{s} \\
\leq B A \sum_{l=0}^{\infty} 2^{l s \alpha} \sum_{k=l}^{\infty} 2^{k(\alpha-\delta) s} \Omega_{\varphi}^{r}\left(f, 2^{-k}\right)_{w, p}^{s}\left\{\sum_{k=l}^{\infty} 2^{-k(\alpha-\delta) s /(s-1)}\right\}^{s-1} \\
\leq C \sum_{l=0}^{\infty} 2^{l s \alpha} 2^{-l(\alpha-\delta) s} \sum_{k=l}^{\infty} 2^{k(\alpha-\delta) s} \Omega_{\varphi}^{r}\left(f, 2^{-k}\right)_{w, p}^{s} \\
\quad=C \sum_{k=0}^{\infty} 2^{k(\alpha-\delta) s} \Omega_{\varphi}^{r}\left(f, 2^{-k}\right)_{w, p}^{s} \sum_{l=0}^{k} 2^{l \delta s} \\
\leq L \sum_{k=0}^{\infty} 2^{k \alpha s} \Omega_{\varphi}^{r}\left(f, 2^{-k}\right)_{w, p}^{s} \leq M \int_{0}^{1}\left(t^{-\alpha} \Omega_{\varphi}^{r}(f, t)_{w, p}\right)^{s} \frac{d t}{t}
\end{aligned}
$$

As

$$
\Omega_{\varphi}^{r}(f, t)_{w, p} \leq A K_{r, \varphi}\left(f, t^{r}\right)_{w, p}
$$

we have now

$$
\|f\|_{B_{\alpha, s}^{p}(\varphi, w)}^{*} \leq M\|f\|_{B_{\alpha, s}^{p}(\varphi, w)} .
$$

Using the definition of $K_{r, \varphi}\left(f, t^{r}\right)_{w, p}$ in (3.3), we have for the best $n$th degree approximating polynomial $P_{n}$

$$
K_{r, \varphi}\left(f,(1 / n)^{r}\right)_{w, p} \leq\left\|w\left(f-P_{n}\right)\right\|_{p}+n^{-r}\left\|w \varphi^{r} P_{n}^{(r)}\right\|_{p}
$$

Using Theorem 8.3 .1 of [5], we have

$$
\left\|w \varphi^{r} P_{n}^{(r)}\right\|_{p} \leq M \sum_{k=0}^{n}(k+1)^{(r-1)} E_{k}(f)_{w, p}
$$


This now implies for $s<\infty$ and $r>\delta>\alpha$

$$
\begin{aligned}
\|f\|_{B_{\alpha, s}^{p}(\varphi, w)}^{s} & \leq M\left(E_{0}(f)_{w, p}^{s}+\sum_{m=0}^{\infty} 2^{m \alpha s} K_{r, \varphi}\left(f, 2^{-m r}\right)_{w, p}^{s}\right) \\
& \leq M_{1}\left[\sum_{m=0}^{\infty} 2^{m \alpha s} E_{2^{m}}(f)_{w, p}^{s}+\sum_{m=0}^{\infty} 2^{m(\alpha-r) s}\left\{\sum_{\nu=0}^{m} 2^{\nu r} E_{2^{\nu}}(f)_{w, p}\right\}^{s}\right] \\
& \leq M_{2}\left[\sum_{m=0}^{\infty} 2^{m \alpha s} E_{2^{m}}(f)_{w, p}^{s}+\sum_{m=0}^{\infty} 2^{m \alpha s} 2^{-m \delta s} \sum_{\nu=0}^{n} 2^{\delta \nu s} E_{2^{\nu}}(f)_{w, p}^{s}\right] \\
& \leq M_{2}\left[\sum_{m=0}^{\infty} 2^{m \alpha s} E_{2^{m}}(f)_{w, p}^{s}+\sum_{\nu=0}^{\infty} 2^{\delta \nu s} E_{2^{\nu}}(f)_{w, p}^{s} \sum_{m=\nu}^{\infty} 2^{m(\alpha-\delta) s}\right] \\
& \leq M_{3} \sum_{m=0}^{\infty} 2^{m \alpha s} E_{2^{m}}(f)_{w, p}^{s} \leq M_{4}\left(\|f\|_{B_{\alpha, s}^{p}(\varphi, w)}^{* *}\right)^{s},
\end{aligned}
$$

and this completes the proof of our theorem.

4. Best algebraic polynomial approximation on polytopes. A polytope in $R^{d}$ is the convex hull of finitely many points. A simple polytope $S$ in $R^{d}$ is a polytope with an interior point in $R^{d}$ having each vertex joined to the other vertices by exactly $d$ edges. For a simple polytope $S$ the relation between certain moduli of smoothness $\omega_{S}^{r}(f, t)_{p}$, defined in [5, Chapter 12], and best algebraic polynomial approximation is given by

$$
E_{n}(f)_{L_{p}(S)} \leq M\left(\omega_{S}^{r}(f, 1 / n)_{p}+n^{-r}\|f\|_{L_{p}(S)}\right), \quad n \geq 1,
$$

and

$$
\omega_{S}^{r}(f, t)_{p} \leq M t^{r} \sum_{0 \leq k \leq 1 / t}(k+1)^{r-1} E_{k}(f)_{L_{p}(S)}
$$

where

$$
E_{n}(f)_{L_{p}(S)}=\inf \left(\|f-P\|_{L_{p}(S)} ; \operatorname{deg} P<n\right)
$$

and where the degree of the algebraic polynomial $P$ could be understood as either the maximum degree in each variable or the sum of the degrees in all variables.

Following almost word for word the considerations of $\S 2$, we have

THEOREM 4.1. For a simple polytope $S$, for $1 \leq p \leq \infty, 1 \leq q \leq \infty$ and $0<\alpha<r$ the norms

$$
\|f\|_{B_{\alpha, q}^{p}(S)}=\|f\|_{L_{p}(S)}+\left\|t^{-\alpha} \omega_{S}^{r}(f, t)_{p}\right\|_{L_{q}^{*}}
$$

and

$$
\|f\|_{B_{\alpha, q}^{p}(S)}^{*}=\left\|(n+1)^{\alpha} E_{n}(F)_{L_{p}(S)}\right\|_{l_{q}^{*}}
$$

are equivalent.

5. Best weighted polynomial approximation in $L_{p}(R)$. Relations between best weighted approximation in $L_{p}(R)$, the $K$ functionals and moduli of smoothness were investigated for Freud's weights by Freud and Mhaskar and in [5, Chapter 
11]. We can now easily obtain an analogue of the results in earlier sections. We concentrate on the weights given by

$$
W_{\lambda}(x)=\exp \left(-|x|^{\lambda}\right), \quad \lambda>1 .
$$

The best polynomial approximation is given by

$$
E_{n}(f)_{W_{\lambda, p}}=\inf \left(\left\|W_{\lambda}(f-P)\right\|_{L_{p}(R)} ; \operatorname{deg} P<n\right) .
$$

The corresponding $K$-functional is given by

$$
K_{r}\left(f, t^{r}\right)_{W_{\lambda, p}}=\inf _{g^{(r-1)} \in \text { A.C.loc }}\left(\left\|W_{\lambda}(f-g)\right\|_{L_{p}(R)}+t^{r}\left\|W_{\lambda} g^{(r)}\right\|_{L_{p}(R)}\right) .
$$

The corresponding main part modulus is given by

$$
\Omega_{r}(f, t)_{W_{\lambda, p}}=\sup _{0<h \leq t}\left\|W_{\lambda} \Delta_{h}^{r} f\right\|_{L_{p}\left[-h^{*}, h^{*}\right]}, \quad h^{*}=h^{1 /(1-\lambda)} .
$$

We can now state and prove the following theorem.

THEOREM 5.1. For $\lambda>1,0<\alpha<r, 1 \leq s \leq \infty$ and $1 \leq p \leq \infty$ the norms

$$
\begin{gathered}
\|f\|_{B_{\alpha, s}^{p}\left(W_{\lambda}\right)}=\left\|f W_{\lambda}\right\|_{L_{p}(R)}+\left\|t^{-\alpha} K_{r}\left(f, t^{r}\right)_{W_{\lambda, p}}\right\|_{L_{s}^{*}}, \\
\|f\|_{B_{\alpha, s}^{p}\left(W_{\lambda}\right)}^{*}=\left\|f W_{\lambda}\right\|_{L_{p}(R)}+\left\|t^{-\alpha} \Omega_{r}(f, t)_{W_{\lambda, p}}\right\|_{L_{s}^{*}},
\end{gathered}
$$

and

are equivalent.

$$
\|f\|_{B_{\alpha, s}^{p}\left(W_{\lambda}\right)}^{* *}=\left\|(n+1)^{\alpha(1-(1 / \lambda))} E_{n}(f)_{W_{\lambda, p}}\right\|_{l_{s}^{*}}
$$

PROOF. We recall [5, Theorem 11.2.5] that

$$
C^{-1} \Omega_{r}(f, t)_{W_{\lambda, p}} \leq K_{r}\left(f, t^{r}\right)_{W_{\lambda, p}} \leq C \int_{0}^{t}\left(\Omega_{r}(f, \tau)_{W_{\lambda, p}} / \tau\right) d t+C e^{-c / t}\left\|W_{\lambda} f\right\|_{L_{p}(R)}
$$

for some $C>0$ and $c>0$. We further recall the results of Freud and Mhaskar with $q_{n}=(n / \lambda)^{1 / \lambda}$ (for $\left.W=W_{\lambda}\right)$ (see [5, Theorem 11.3.2]) that

$$
E_{n}(f)_{W_{\lambda, p}} \leq C K_{r}\left(f, n^{r((1 / \lambda)-1)}\right)_{W_{\lambda, p}}, \quad n>r
$$

and

$$
K_{r}\left(f, n^{r((1 / \lambda)-1)}\right)_{W_{\lambda, p}} \leq C n^{r((1 / \lambda)-1)} \sum_{k=0}^{n} k^{r(1-(1 / \lambda))-1} E_{k}(f)_{W_{\lambda, p}} .
$$

In some ways the proof is easier here than that of Theorem 5.3 as we have (5.7) and we did not have a corresponding expression for general weights of class $J_{p}^{*}$. (Theorem 6.2 .3 of [5] will yield a slightly more convenient inequality for Jacobi weights.) Therefore, arguments analogous to those using (3.10) and (3.11) in the proof of Theorem 3.1 are redundant as we use (5.7). The other part of the proof follows the proof of Theorems 2.1 and 3.1. For instance, we have

$$
\begin{aligned}
& \sum_{n=0}^{\infty}(n+1)^{\alpha(1-(1 / \lambda)) s} E_{n}(f)_{W_{\lambda, p}}^{s} \leq C_{1} \sum_{m=0}^{\infty} 2^{m \alpha(1-(1 / \lambda)) s} E_{2^{m}}(f)_{W_{\lambda, p}}^{s} \\
& \quad \leq C_{2}\left(\left\|W_{\lambda} f\right\|_{p}^{s}+\sum_{m=0}^{\infty} 2^{m \alpha(\lambda-1) s / \lambda} K_{r}\left(f, 2^{m r(1-\lambda) / \lambda}\right)\right)_{W_{\lambda, p}}^{s} \\
& \quad \leq C_{3}\left(\left\|W_{\lambda} f\right\|_{p}^{s}+\int_{0}^{t_{0}}\left(t^{-\alpha} K_{r}(f, t)_{W_{\lambda, p}}\right)^{s} \frac{d t}{t}\right),
\end{aligned}
$$


which implies $\|f\|_{B_{\alpha, s}^{p}\left(W_{\lambda}\right)}^{* *} \leq M\|f\|_{B_{\alpha, s}^{p}\left(W_{\lambda}\right)}$. We can also write

$$
\begin{aligned}
\left\|W_{\lambda} f\right\|_{p}^{s}+\int_{0}^{\infty} t^{-\alpha s} K_{r}\left(f, t^{r}\right)_{W_{\lambda, p}}^{s} \frac{d t}{t} \\
\leq C_{1}\left(E_{0}(f)_{W_{\lambda, p}^{s}}+\sum_{l=0}^{\infty} 2^{l s(1-(1 / \lambda)) \alpha} K_{r}\left(f, 2^{r l((1 / \lambda)-1)}\right)_{W_{\lambda, p}^{s}}\right) \\
\leq C_{2} \sum_{l=0}^{\infty} 2^{l s(1-(1 / \lambda))(\alpha-r)}\left\{\sum_{\nu=0}^{l} 2^{\nu r(1-(1 / \lambda))} E_{2^{\nu}}(f)_{W_{\lambda, p}}\right\}^{s} \\
\leq C_{3}\left(\|f\|_{B_{\alpha, s}^{p}\left(W_{\lambda}\right)}^{* *} \cdot \quad \square\right.
\end{aligned}
$$

\section{REFERENCES}

1. P. L. Butzer and H. Berens, Semi-groups of operators and approximation, Springer-Verlag, 1967.

2. P. L. Butzer and K. Scherer, Approximationprozesse und interpolationsmethoden, Hochschulskripten 826/826a, Bibliograph. Institute, 1968.

3. R. A. DeVore, S. D. Riemenschneider and R. C. Sharpley, Weak interpolation in Banach spaces, J. Functional Anal. 33 (1979), 58-94.

4. R. DeVore and V. Popov, Interpolation and nonlinear approximation, Proc. Conf. Lund, 1986, Lecture Notes in Math., 1988, Springer-Verlag, 191-205.

5. Z. Ditzian and V. Totik, Moduli of smoothness, Springer-Verlag, 1987.

6. G. I. Sunouchi, Derivative of a trigonometric polynomial of best approximation, Abstract Spaces and Approximation (P. L. Butzer and B. Sz. Nagy, eds.), Proc. Conf. Oberwolfach, July 18-27, 1968, Birkhäuser-Verlag, 1969, pp. 233-241.

Department of Mathematics, University of Alberta, Edmonton, Alberta, CANADA T6G 2G1

Bolyai Institute, Altila Jósef University, Szeged Aradi U., tere 1, 6720 HUNGARY 